\title{
Analytical Study of Precision Optical Glass Surface and Its Effect on Some Polarimetric Parameters
}

\author{
Naglaa Aboe-Ella Mahmoud \\ Length and Engineering Precision, National Institute for Standards (NIS), Giza, Egypt \\ Email: naglaamahmoud2006@yahoo.com
}

How to cite this paper: Mahmoud, N.A.-E. (2022) Analytical Study of Precision Optical Glass Surface and Its Effect on Some Polarimetric Parameters. Journal of Surface Engineered Materials and Advanced Technology, 12, 14-21.

https://doi.org/10.4236/jsemat.2022.121002

Received: October 23, 2021

Accepted: January 10, 2022

Published: January 13, 2022

Copyright $\odot 2022$ by author(s) and Scientific Research Publishing Inc. This work is licensed under the Creative Commons Attribution International License (CC BY 4.0).

http://creativecommons.org/licenses/by/4.0/

\begin{abstract}
The performance of many optical glass elements depends on the structure of the surface. The high refractive index of flint glass is advantageous in constructing some optical elements (lenses, prisms, beam splitters, ...). Also, high achromaticity and size reduction in oblique incidence TIR (total internal reflection) phase retarders require high-index glass. The present work is interested in studying the optical properties of the thin surface layer formed on TIR rhomb retarder at different wavelengths to indicate the extent to which this surface layer affects the performance of this TIR rhomb retarder and show how to overcome this effect.
\end{abstract}

\section{Keywords}

Optical Glass, Ellipsometry, Oblique Incidence Retarder, Surface Layer and Retardance

\section{Introduction}

The optical glass used in manufacturing optical elements or devices is subjected to mechanical polishing, chemical etching, or other processes to improve the quality of its surfaces. The performance of many optical glass elements depends on the structure of the surface. Thus, a clean and smooth surface with a similar composition to that of the bulk glass is essentially required in many optical elements. However, these requirements cannot be always fulfilled, particularly because of surface treatment, glass degradation, environmental effects, and other factors that lead to the formation of surface layer. Single homogeneous surface film model and effective medium model describing nano roughness of the surface are usually considered in studying the formed surface layers [1] [2] [3] [4]. 
Quality control of a functional surface of optical glasses must be precise to give an optimally functional state, enough rapid and economic. There are many methods for measuring the surface roughness of glasses such as image processing, microscopes, stylus type instruments, profile tracing instruments, etc., [5]. The contact methods for the determination of roughness are undesirable because they are destructive and let prints on the tested surface. Ellipsometric technique can be used to determine the optical constants, the surface thickness and roughness of the bulk optical glass. It has several advantages over other methods. Among these are the simplicity of measurement and the ease of sample preparation. Besides, it is non-destructive and requires only a very small sample size.

Ellipsometry can be conveniently divided into two parts, the first is the measurement technique for determining $\psi$ and $\Delta$, which describe the change in amplitude and phase in the reflected wave. The second is the theory required to relate the optical parameters of the thin film formed on the substrate, (the refractive index $n$, the extinction coefficient $k$, and the thickness $d$ ) to the measured values of $\psi$ and $\Delta$.

Figure 1, represents a thin film of thickness $d$ and optical constants $n_{l}, k_{l}$ over the substrate. $E_{P}$ and $E_{s}$ are the components of the incident field in the plane of incidence and perpendicular to the plane of incidence. The components of the reflected field are $R_{P}$ and $R_{S}$.

For the incident and the reflected fields, suppressing the propagation factor,

$$
\begin{aligned}
& E_{P}=E_{0 P} \mathrm{e}^{i \alpha_{p}}, \\
& E_{s}=E_{0 s} \mathrm{e}^{i \alpha_{s}}, \\
& R_{P}=R_{0 P} \mathrm{e}^{i \beta_{p}}, \\
& R_{s}=R_{0 s} \mathrm{e}^{i \beta_{s}},
\end{aligned}
$$

where $\left(\alpha_{p}, \alpha_{s}\right)$ and $\left(\beta_{p}, \beta_{s}\right)$ are the phases of the incident and reflected components, introducing the complex reflection coefficients $\rho_{p}, \rho_{s}$ relating the reflected field components to the incident field components.

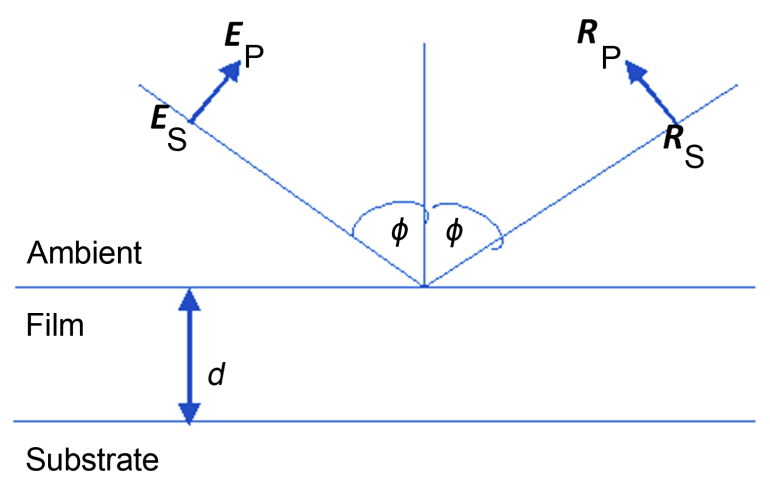

Figure 1. Film/substrate structure. $d$ is the film thickness, $p$ and $s$ are the parallel and perpendicular components of the incident and reflected light, and $\Phi$ is the angle of incidence. 


$$
\begin{gathered}
R_{p}=\rho_{p} E_{p}, \\
R_{s}=\rho_{s} E_{s} .
\end{gathered}
$$

Defining a complex relative amplitude attenuation $\rho$ as

$$
\rho=\rho_{p} / \rho_{\mathrm{s}}
$$

Then,

$$
\rho=\left[\left(R_{0 p} / E_{0 P}\right) /\left(E_{0 s} / E_{0 S}\right)\right] \mathrm{e}^{i(\beta-\alpha)}
$$

where $\alpha=\alpha_{p}-\alpha_{s}$ and $\beta=\beta_{p}-\beta_{s}$.

We can express $\Delta=(\beta-\alpha)$ and $\tan \psi=\left(R_{0 p} / E_{0 P}\right) /\left(E_{0 s} / E_{0 S}\right)$.

So,

$$
\rho=\tan \psi \mathrm{e}^{i \Delta}=f(n, k, d)
$$

which is called the fundamental equation of ellipsometry. By finding the values of $\psi$ and $\Delta$ using ellipsometric analysis at a definite angle of incidence $\Phi$ and the value of $n_{g}$ (the refractive index of the glass) known in advance, the problem seems to relate these values to $n, k$, and $d[6]$.

The present work was initiated by the fact that on calibrating an oblique-incidence $\lambda / 4$ phase retarder [7], Figure 2 at wavelength $(589 \mathrm{~nm})$, it was found that the device provided a retardance of $87^{\circ}$, i.e., $3^{\circ}$ less than the theoretically calculated value.

This difference could be attributed to several factors including error in the external angle of incidence, birefringence in the bulk glass, a manufacturing error in the acute angle $\alpha$ or formation of surface layers on the reflecting surfaces. This device belongs to a class of total internal (TIR) phase retarder known as oblique-incidence retarders. In this class, the angle of (TIR) $\theta$ are not of fixed value but varies with the refractive index $n$ of the used glass (or equivalently, with the wavelength $\lambda$ of the incident light) [8] [9].

Such that,

$$
\theta=\alpha+t
$$

And

$$
t=\sin ^{-1}(\sin i / n)
$$

where $\alpha$ is the angle between the entrance face and the reflecting surface, $i$ is the angles of incidence and $t$ is the angle of refraction. From the previous equation, we notice that, for the same value of incidence $i$, the angle of $t$ will vary with $n$ (or with $\lambda$ ). Thus, $\theta$ is no longer of fixed value and the retardance is expressed as:



Figure 2. Oblique incidence $4 / \lambda$ phase retarder, $n_{g}=1.70002$ at $589 \mathrm{~nm}$. 


$$
\delta=f(n, \theta)
$$

where, $\theta=f_{1}(n)$ and $n=f_{2}(\lambda)$.

Present work is interested in studying the optical properties of the thin surface layer formed on this TIR rhomb retarder which is made of flint glass $\left(n_{g}=\right.$ 1.70002 at $589 \mathrm{~nm})$ at spectral wavelengths from $(400-800) \mathrm{nm}$ in steps $100 \mathrm{~nm}$ and will study the effect of this formed layer on the performance of this TIR rhomb retarder. The effective medium approximation can then be used to analyze the measured ellipsometric values assuming the effective medium layer to consist of a physical mixture interface of air and glass.

\section{Experimental Work}

We studied the surface layer formed on one of the two reflecting faces of TIR rhomb retarder shown in Figure 2, which is made of flint glass $\left(n_{g}=1.70002\right.$ at $589 \mathrm{~nm}$ ). The measuring equipment used is Spectroscopic ellipsometer PHE103 (Angstrom Advanced Inc.). It is an automated version supported by analyzing software. The sample is placed on a motorized stage that is controlled by the software and enables fine adjustment of incidence angle and the ellipsometric angles are measured with accuracy $\pm 0.01^{\circ}$. A schematic diagram of the ellipsometer is presented in Figure 3 which consists of a light source, polarizer, analyzer, a quarter-phase plate (compensator), $\Phi$ is the angle of incidence and detecting system. The angle of incidence $\Phi$ was set at $\left(\Phi=60^{\circ}, 65^{\circ}, 70^{\circ}\right)$. The measurements were carried out in the spectral wavelengths from $(400-800) \mathrm{nm}$ in steps $100 \mathrm{~nm}$ and temperature of $(20 \pm 0.05)^{\circ} \mathrm{C}$.

\section{Results}

The values of the ellipsometric parameters $\psi$ and $\Delta$ according to the angles of incidence within the interval $60^{\circ}$ and $70^{\circ}$ are gathered in Table 1 and Figure 4 and Figure 5.

The values obtained from Table 1 allow drawing up Table 2 and Figure 6 for the values of $n_{6}$ (the refractive index of the layer formed on the surface of the glass) versus the spectral wavelengths from $(400-800) \mathrm{nm}$ in steps $100 \mathrm{~nm}$ for different angles of incidence.

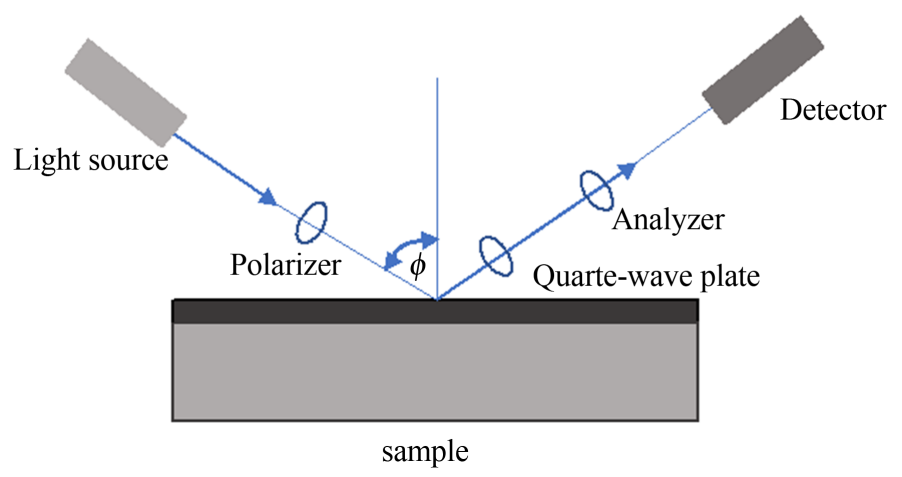

Figure 3. Ellipsometric schematic diagram. 


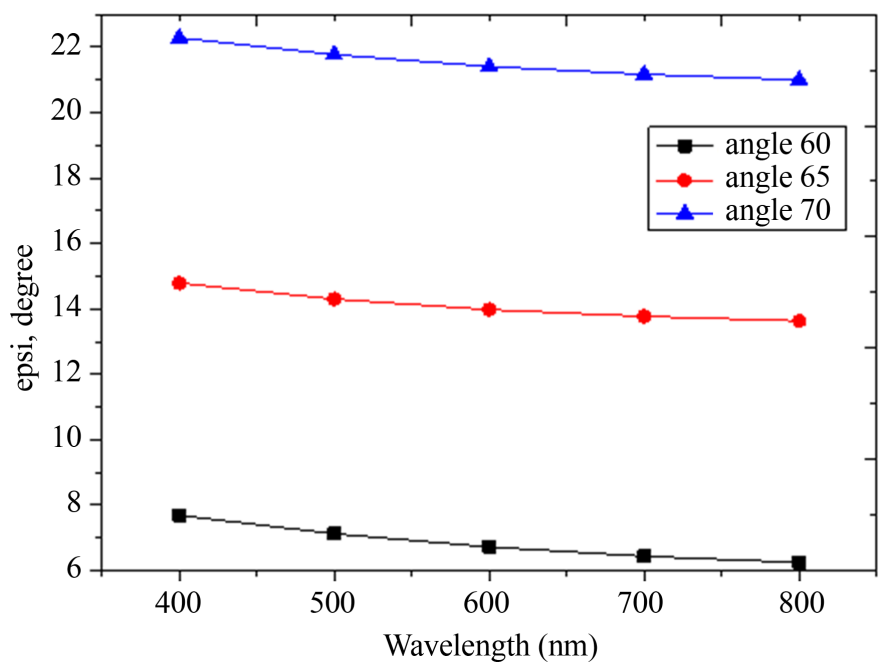

Figure 4. The variation of $\Psi$ versus the wavelength for different angles of incidence.

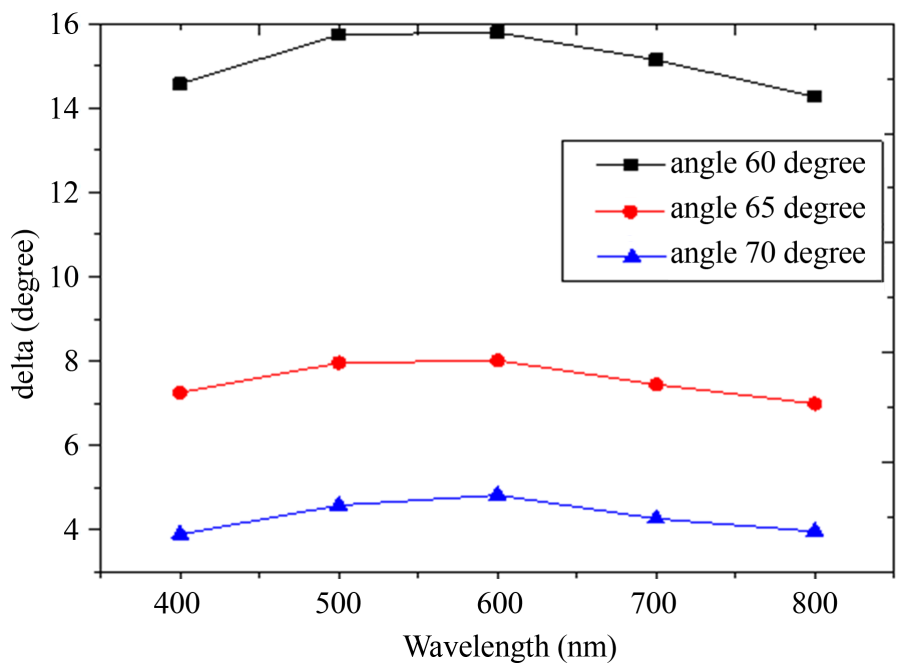

Figure 5. The variation of $\Delta$ versus the wavelength for different angles of incidence.



Figure 6. The variation of $n_{f}$ versus the wavelength for different angles of incidence. 
Table 1. Values of $\psi$ and $\Delta$ versus the wavelengths for different values of angle of incidence.

\begin{tabular}{ccccccc}
\hline \multirow{2}{*}{$\begin{array}{c}\text { Wavelength, } \\
\lambda \text { nm }\end{array}$} & \multicolumn{2}{c}{$\Phi=60^{\circ}$} & \multicolumn{2}{c}{$\Phi=65^{\circ}$} & \multicolumn{2}{c}{$\Phi=70^{\circ}$} \\
\cline { 2 - 7 } & $\begin{array}{c}\Psi \\
\left.{ }^{\circ}\right)\end{array}$ & $\begin{array}{c}\Delta \\
\left(^{\circ}\right)\end{array}$ & $\begin{array}{c}\Psi \\
\left.{ }^{\circ}\right)\end{array}$ & $\begin{array}{c}\Delta \\
\left(^{\circ}\right)\end{array}$ & $\begin{array}{c}\left.{ }^{\circ}\right) \\
\left(^{\circ}\right)\end{array}$ \\
\hline 400 & 7.69 & 13.57 & 14.79 & 7.26 & 22.28 & 3.9 \\
500 & 7.13 & 15.74 & 14.30 & 7.96 & 21.77 & 4.59 \\
600 & 6.73 & 15.79 & 13.98 & 8.02 & 21.41 & 4.83 \\
700 & 6.44 & 15.14 & 13.77 & 7.45 & 21.17 & 4.27 \\
800 & 6.25 & 14.27 & 13.63 & 7.00 & 21.00 & 3.97 \\
\hline
\end{tabular}

Table 2. Values of the refractive index versus the wavelengths for different angles of incidence.

\begin{tabular}{cccc}
\hline $\begin{array}{c}\text { Wavelength, } \\
\lambda \mathrm{nm}\end{array}$ & $\begin{array}{c}n_{f} \\
\left(\Phi=60^{\circ}\right)\end{array}$ & $\begin{array}{c}n_{f} \\
\left(\Phi=65^{\circ}\right)\end{array}$ & $\begin{array}{c}n_{f} \\
\left(\Phi=70^{\circ}\right)\end{array}$ \\
\hline 400 & 1.6440 & 1.6566 & 1.6466 \\
500 & 1.6125 & 1.6289 & 1.6489 \\
600 & 1.5975 & 1.6134 & 1.5934 \\
700 & 1.5890 & 1.5915 & 1.5899 \\
800 & 1.5834 & 1.5945 & 1.5802 \\
\hline
\end{tabular}

The values of the thickness of the formed layer $d_{f}$ according to the angles of incidence within the interval $60^{\circ}$ and $70^{\circ}$ showed in Table 3 .

Table 4 and Figure 7 show the differences between the refractive index for the formed layer $n_{f}$ at the angle of incidence $60^{\circ}$ and that of the glass substrate $n_{s}$.

From the analytical studying of the glass surface using ellipsometric method, The average $n_{f}$ for $60^{\circ}, 65^{\circ}$ and $70^{\circ}$ angles of incidence at $589 \mathrm{~nm}$ wavelength equals 1.6000 and $d_{f}$ was extracted from Table 3 equals $122 \mathrm{~nm}$.

\section{Discussion}

The experimental results showed a reduction of approximately 0.10 than the refractive index of the glass of the rhomb for all wavelengths. The main factor for this reduction in the formation of a surface layer on the sides of the rhomb, which in turn, explains the large reduction in the retardance value of the rhomb $\left(-3^{\circ}\right)$ during the calibration of the rhomb retarder. Other factors are the polishing effects and internal stresses [10]. In many optical measurements the surface layer, usually present on most glasses, is neglected either because it does not affect the measurement, or its effect is negligible. However, for measurements made using polarized light, particularly in ellipsometric measurements, the homogeneity of the studied surface is extremely important [11].

According to the relation, 


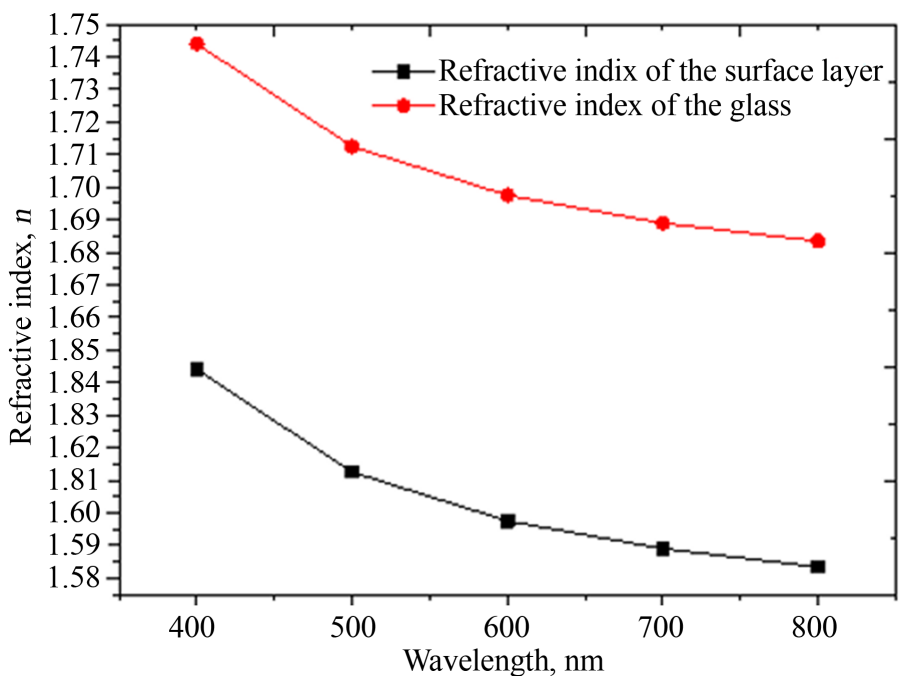

Figure 7. The differences between the refractive index for the formed layer $n_{f}$ at the angle of incidence $60^{\circ}$ and that of the glass substrate ns.

Table 3. Values of the thickness of the formed layer versus the angle of incidence.

\begin{tabular}{cc}
\hline Angle of incidence, $\Phi\left(^{\circ}\right)$ & $d_{f}(\mathrm{~nm})$ \\
\hline 60 & 121 \\
65 & 120 \\
70 & 125 \\
\hline
\end{tabular}

Table 4. The refractive index of the formed layer and that of the glass versus the wavelength at $\Phi=60^{\circ}$.

\begin{tabular}{ccc}
\hline Wavelength, $\lambda \mathrm{nm}$ & $n_{f}\left(\Phi=60^{\circ}\right)$ & $n_{s}$ \\
\hline 400 & 1.6440 & 1.7440 \\
500 & 1.6125 & 1.7125 \\
600 & 1.5975 & 1.6975 \\
700 & 1.5890 & 1.6890 \\
800 & 1.5834 & 1.6834 \\
\hline
\end{tabular}

$$
\theta=\alpha+t=\alpha+\sin ^{-1}(\sin i / n)
$$

where $i$ and $t$ are the angles of incidence and refraction on the entrance face.

We notice that the angle $t$ varies with $n$. Thus, $\theta$ is no longer of fixed value and in this case the retardance is expressed as

$$
\delta=f(n, \theta)
$$

where $\theta=f_{1}(n)$ and $n=f_{2}(\lambda)$.

The retardance of our rhomb could be adjusted to a value close to $90^{\circ}$ by a small variation in the external angle of incidence $i$ [12]. We note that variations in the external angle of incidence $i$ could be compensated with the variation of $n$. Thus, the device retardance can be adjusted will by simply changing angle $i$. 


\section{Conclusion}

This work describes the use of ellipsometry as a precise and accurate technique to test and monitor changes of surfaces of optical components because of the manufacturing or by environmental surroundings, which affects some optical measurements such as the retardance value of TIR rhomb retarder, to adjust and make corrections in the result values.

\section{Conflicts of Interest}

The author declares no conflicts of interest regarding the publication of this paper.

\section{References}

[1] Mazza, M., Farnoux, B., Samuel, F., Sella, C. and Trocellier, P. (1993) Effect of Mechanical Polishing on Surface Structure of Glasses Studied by Grazing Angle Neutron Reflectometry. Optics Communications, 100, 220-230. https://doi.org/10.1016/0030-4018(93)90584-R

[2] Ledieu, A., Devreux, F. and Barboux, P. (2004) Monte Carlo Simulation of Borosilicate Glass Corrosion: Predictions for Morphology and Kinetics. Journal of Non-Crystalline Solids, 345-346, 715-719. https://doi.org/10.1016/j.jnoncrysol.2004.08.152

[3] Ziemath, E.C. (1998) Degradation of the Surface of a Metasilicate Glass Due to Atmosphere Moisture. Química Nova, 21, 356-360. https://doi.org/10.1590/S0100-40421998000300021

[4] Fujiwara, F., Koh, J., Rovira, P.I. and Collins, R.W. (2000) Assessment of Effective Medium Theories in the Analysis of Nucleation and Microscopic Surface Roughness Evolution for Semiconductor Thin Films. Physical Review B, 61, 10832-10844. https://doi.org/10.1103/PhysRevB.61.10832

[5] Çolak, O., Kubanoelu, C. and Kayacan, M.C. (2007) Milling Surface Roughness Prediction Using Evolutionary Programming Methods. Materials \& Design, 28, 657. https://doi.org/10.1016/j.matdes.2005.07.004

[6] Goldstein, D. (2003) Polarized Light. Second Edition, Marcel Dekker, New York.

[7] Mahmoud, N.A. (2007) Studies on Some Optical Polarization Elements and Measurements. M.Sc. Thesis, Faculty of Science, Cairo University, Cairo.

[8] Nagib, N.N. and Khodier, S.A. (1995) Optimization of a Rhomb-Type Quarter-Wave Phase Retarder. Applied Optics, 34, 2927-2930. https://doi.org/10.1364/AO.34.002927

[9] Nagib, N.N. (1997) Theory of Oblique Phase Retarders. Applied Optics, 36, 1547-1552. https://doi.org/10.1364/AO.36.001547

[10] King, R.J. (1966) Quarter-Wave Retardation System Based on the Fresnel Rhomb Principle. Journal of Scientific Instruments, 43, 617-622. https://doi.org/10.1088/0950-7671/43/9/301

[11] Bennett, J.M. and King, R.J. (1970) Effect of Polishing Technique on the Roughness and Residual Film on Fused Quartz Optical Flats. Applied Optics, 9, 236-238. https://doi.org/10.1364/AO.9.000236

[12] Nagib, N.N., Mahmoud, N.A. and Barhawi, M.S. (2018) Realization and Calibration of an Oblique-Incidence Rhomb Retarder of Extreme Retardance Stability. Measurement, 124, 156-158. https://doi.org/10.1016/j.measurement.2018.04.032 